\title{
Does dedicated pre-abortion contraception counselling help to improve post-abortion contraception uptake?
}

\author{
Ahmed S Yassin, Diane Cordwell
}

\begin{abstract}
Objective Many studies have shown a disappointing periabortion contraceptive uptake. This study investigated whether the provision of dedicated and targeted contraception counselling at the pre-abortion assessment visit can improve the post-abortion contraception uptake.

Methods The study comprised a 3-month prospective reaudit of the abortion clinic.

Results Of the 104 women seen during the re-audit period, 96\% received post-abortion contraception. The majority $(73 \%)$ of the women chose and received one of the less user-dependent contraceptive methods such as intrauterine contraceptive devices, the intrauterine contraceptive system, the progestogen injectable and subdermal implants.
\end{abstract}

Conclusion It was found that the provision of targeted contraception counselling by a dedicated team during the pre-abortion assessment visit can dramatically improve post-abortion contraception uptake.

J Fam Plann Reprod Health Care 2005; 31(2): 115-116 (Accepted 22 November 2005)

\section{Key message points \\ - The majority of women requesting first-time or repeat abortion use no contraception or use a compliance- dependent method such as condoms or pills. \\ - The provision of targeted contraceptive counselling by a dedicated team during the pre-abortion assessment can dramatically improve the post-abortion contraception uptake.}

\section{Introduction}

The number of women requesting abortion is rising. ${ }^{1}$ The majority of these women either use no contraception or use a user-dependent method such as the oral contraceptive pill (OCP) or condoms. ${ }^{2}$ The guidelines for induced abortion issued by the Royal College of Obstetricians and Gynaecologists (RCOG) emphasise the importance of contraception counselling as an essential part of abortion services. ${ }^{3}$ Many abortion clinics discuss contraception at the pre-abortion assessment and rely on the post-abortion follow-up [which can be at the abortion clinic, the gynaecology clinic or the general practice and family planning clinics (FPCs)] for the provision of contraception. This lack of clarity of role, combined with the poor attendance for follow-up after abortion, was shown to result in a disappointing peri-abortion contraception uptake. ${ }^{2}$

The Women's Unit, Stepping Hill Hospital, Stockport, UK Ahmed S Yassin, DFFP, MRCOG, Specialist Registrar in Obstetrics and Gynaecology and Instructor in Family Planning

Diane Cordwell, RGN, RSCN, Family Planning Nurse

Correspondence to: Dr Ahmed S Yassin, Women's Health Unit, Burnley General Hospital, Casterton Avenue, Burnley BB10 2PQ, UK. E-mail: ASAY5960@aol.com

\section{Background}

In 2001, our abortion clinic, which provides surgical termination of pregnancy (STOP) only, was staffed by a traditional abortion counsellor, a gynaecologist and general nurses. It was felt that the clinic, with the current level of staffing and expertise base, offered an unsatisfactory contraception service to women requesting abortion. A retrospective audit for the year 2000-2001 was done primarily to identify the level of post-abortion contraception uptake. The audit showed that only $40 \%$ of the 422 women seen at the clinic received post-abortion contraception, mainly in the form of OCPs, condoms and Depo-Provera ${ }^{\circledR}$ injections.

In an effort to improve the post-abortion contraception uptake we recruited to our pre-abortion assessment clinic two experienced family planning nurses who had undergone additional training in counselling. Our medical team has undergone training in all aspects of family planning, has obtained letters of competence from the Faculty of Family Planning and Reproductive Health Care, and is involved in running FPCs on a regular basis.

Dedicated contraceptive counselling became an integral part of every consultation at the abortion clinic. All methods of contraception were discussed equally, and during counselling we emphasised the superior effectiveness of the intrauterine contraceptive device (IUD), intrauterine contraceptive system (IUS), DepoProvera injections and subdermal implants since they are less user-dependent methods when compared to OCPs and barrier methods.

As in any mainstream FPC, the woman was seen first by the family planning nurse who, in addition to providing abortion counselling, also provided contraception counselling and handed out information leaflets. The woman was than seen by the medical team who, in addition to performing a pre-abortion assessment, offered the opportunity for a full and detailed discussion about the chosen contraceptive method. It was made clear to the clients that the abortion clinic's agreeing to offer abortion was not affected in any way by the woman's acceptance of any particular contraception method.

This re-audit was conducted to find out whether the above mentioned measures have improved post-abortion contraception uptake at the abortion clinic.

\section{Methods}

This was a prospective re-audit of the abortion clinic during a 3-month period between September and December 2003. Using Microsoft Excel, we collected data from all the women who attended the clinic during this period. The following data were collected: the demographic characteristics of the women, details of any previous terminations of pregnancy, details of previous contraception and the reason for failure, and the contraceptive method chosen as a result of counselling. Ninety-eight $(94.2 \%)$ women underwent an abdominal or transvaginal scan. All the women had STOP under general anaesthesia and received prophylactic antibiotics as per the RCOG protocol. ${ }^{3}$ OCPs were given at the assessment clinic to start the next day after the procedure. IUDs, the IUS and progestogen implants were inserted at time of the procedure, and Depo-Provera injections were given in the postoperative recovery room. 
ARTICLE

Table 1 Contraception usage and reasons for contraceptive failure

\begin{tabular}{ll}
\hline $\begin{array}{l}\text { Contraceptive method/reasons } \\
\text { for failure }\end{array}$ & $\begin{array}{l}\text { Women }[n(\%)] \\
(n=104)\end{array}$ \\
\hline No contraception & $57(54.8)$ \\
Rhythm method & $1(0.9)$ \\
Condom & $37(35.6)$ \\
$\quad$ Split & 22 \\
Came off & 2 \\
$\quad$ Unexplained & 13 \\
Oral contraceptive pill & $9(8.6)$ \\
$\quad$ Missed pill & 5 \\
Vomiting & 1 \\
$\quad 3 n$ Xnplained & 3 \\
Progestogen injection & 0 \\
Intrauterine device & 0 \\
\hline
\end{tabular}

\section{Results}

A total of 104 women were seen during the re-audit period. The median age was 26 (range, 15-41) years; $15(14.4 \%)$ women were aged $>35$ years and $33(31.7 \%)$ were aged $<20$ years. Table 1 shows the contraceptive method used by the women and the reason for contraceptive failure. Twentythree $(22.1 \%)$ woman had a previous induced abortion. Fifteen $(65.2 \%)$ of them used no contraception and eight $(34.8 \%)$ used condoms. Coincidentally, four $(4.1 \%)$ women were found to have pregnancy failure, which was confirmed by serial scans at the early pregnancy assessment unit. The remaining 100 women received contraception counselling. Ninety-six $(96 \%)$ women received contraception. Table 2 shows the contraceptive method chosen and received by women following contraception counselling compared to the group of women in the 2000-2001 audit.

\section{Discussion}

The results of this re-audit confirmed the findings of other studies and audits, namely that the majority of women requesting first-time or repeat abortion use no contraception or use a user-dependent method such as condoms or the OCP. ${ }^{2,4}$ Although both the condom and OCP methods have a low Pearl index, they have much higher failure rates in practice due to user error, improper use or accidents. 5

The incidence of repeat abortion in our study was $22.1 \%$ in comparison to the figure of $27 \%$ quoted by another UK study. 5 Garg et al. suggested that the use of alternative forms of contraception, such as the IUD and progestogen injection, could prevent up to $68 \%$ of repeat abortions in their series. They concluded that a favourable impact on the incidence of repeat abortion could be achieved by emphasising that OCPs require user compliance and that barrier methods used alone are less effective. Garg et al. went on to propose a more thorough peri-abortion contraceptive counselling with structured follow-up. ${ }^{4}$

Table 2 Contraceptive methods chosen and received by the women after contraceptive counselling compared to the group of women in the 2000-2001 audit

\begin{tabular}{lll}
\hline Contraceptive method & $\begin{array}{l}2000-2001 \\
\text { audit }(\%) \\
(n=422)\end{array}$ & $\begin{array}{l}2003 \\
\text { re-audit }(\%) \\
(n=100)\end{array}$ \\
\hline No contraception & 60 & 4 \\
Condom & 10 & 3 \\
$\begin{array}{l}\text { Oral contraceptive pill } \\
\text { Progestogen injection } \\
\quad\left(\text { Depo-Provera }{ }^{\circledR}\right)\end{array}$ & 18 & 20 \\
$\begin{array}{l}\text { Progestogen implant } \\
\left(\text { Implanon }{ }^{\circledR} \text { ) }\right.\end{array}$ & 12 & 15 \\
$\begin{array}{l}\text { Intrauterine system } \\
(\text { Mirena }\end{array}$ & 0 & 11 \\
$\begin{array}{l}\text { Intrauterine contraceptive } \\
\text { device }\end{array}$ & 0 & 32 \\
\hline
\end{tabular}

Many of the abortion clinics discuss contraception at the pre-abortion assessment visit but rely on the postabortion follow-up for the provision of the chosen contraceptive method. However, previous studies have shown that after leaving the abortion clinic, many women may not attend for follow-up and indeed may not want to be contacted. ${ }^{6}$ Kumar et al. showed that attendance for follow-up after abortion is poor and there is no clarity of role, with a tendency for health professionals to assume that the next person 'down the line' would deal with the issue of contraception. ${ }^{2}$

Our results demonstrated that $96 \%$ of the women chose and received post-abortion contraception in comparison to only $40 \%$ in the 2000-2001 audit. More importantly, $73 \%$ of these women chose and received one of the less userdependent methods such as IUDs, the IUS, Depo-Provera injections and progestogen implants as a result of targeted counselling that emphasised the superior effectiveness of these methods.

It can be argued that the high uptake of the less userdependent methods during STOP can be explained by the fact that women are likely to be happier with one of these more 'invasive' methods because they will be under general anaesthetic at the time of fitting. However, the uptake of these methods was only $12 \%$ during the 2000-2001 audit when women received poor contraception counselling (i.e. only Depo-Provera) (Table 2).

The findings of this re-audit confirmed our belief that the provision of targeted pre-abortion contraceptive counselling by a dedicated family planning team can be a very effective tool for increasing women's confidence and commitment to having the chosen method of contraception initiated immediately after abortion.

It will be interesting to see, however, if the provision of these less user-dependent methods will reduce the incidence of repeat abortion. This will require follow-up and could form the basis of further work, which will also allow assessment of the women's continuation with their chosen contraceptive methods. It will also be interesting to observe whether a similar contraceptive uptake can be achieved after medical abortion.

\section{Conclusions}

We believe that the provision of targeted contraceptive counselling by a dedicated team at the pre-abortion assessment visit can dramatically improve post-abortion contraception uptake and ensure that every woman presenting at the abortion clinic is confident in having a long-term, highly effective method of contraception that is acceptable to her and can reduce the risk of repeat abortion.

Statements on funding and competing interests

Funding. None identified.

Competing interests. None identified.

References

1 Office for National Statistics. Abortion Statistics 2000. London, UK: Office for National Statistics, 2001.

2 Kumar U, Baraitser P, Morton S, Massil H. Peri-abortion contraception: a qualitative study of users' experiences. J Fam Plann Reprod Health Care 2004; 30: 55-56.

3 Royal College of Obstetricians and Gynaecologists (RCOG). The Care of Women Requesting Induced Abortion. Evidence-based Guideline No. 7. London, UK: RCOG, 2004.

4 Garg M, Singh M, Mansour D. Peri-abortion contraceptive care: can we reduce the incidence of repeat abortion? J Fam Plann Reprod Health Care 2001; 27: 77-80.

5 Lewis C, Wood C, Randall S. Unplanned pregnancy: is contraception failure predictable? Br J Fam Plann 1996; 22: 16-19.

6 Hopwood J, Mallinson EJ, Jones I. There is more to a test than technology - evaluation of testing for chlamydia infection in a charitable sector termination service. Br J Fam Plann 1998; 23: 116-119. 